\title{
A Novel Hierarchical Level Set with AR-Boost for White Matter Lesion Segmentation in Diabetes
}

\author{
Baidya N. Saha, Sriraam Natarajan, Gopi Kota, Christopher T. Whitlow, Donald Bowden, Jasmin Divers, Barry \\ I. Freedman, Joseph A. Maldjian
}

Wake Forest School of Medicine, Winston-Salem, North Carolina, USA, 27157

\{bsaha, snataraj, gkota, cwhitlow, dbowden, jdivers, bfreedma, maldjian\}@wakehealth.edu

\begin{abstract}
Hierarchical as well as coupled level sets are widely used for multilevel image segmentation. However, these tools are successful if the number of levels of an image are known and a careful choice of initialization is performed. We intend a novel hierarchical level set (HLS) followed by an Adaptive Regularized Boosting (AR-Boost) for automatic White Matter Lesion (WML) segmentation from Magnetic Resonance Images. HLS does not need to know the number of levels in an image and HLS is computationally less expensive and more initialization independent than coupled level-sets since HLS doesn't generate redundant regions. We employ an energy functional that minimizes the negative logarithm of variances between the two partitions created by the level set function. HLS uses a level set to partition the image into a number of segments, then applies the level set on all the segments separately to create more segments and the process continues iteratively until all the segments become a nearly homogeneous region (low intensity variance). Then AR-Boost classifies the segments into WML and non-WML classes. The proposed loss function for AR-boost enforces more weight on misclassified samples at each iteration than Adaboost to classify correctly in the next iteration and consequently leads to early convergence. Unlike Adaboost, the user can select optimal weights through cross-validation. Experimental results demonstrate that the proposed method outperforms state-ofthe-art automated white matter lesion segmentation techniques.
\end{abstract}

Keywords-White Matter Lesion Segmentation; Level Set; Boosting; Diabetes

\section{INTRODUCTION}

White Matter Lesions (WML) are thought to reflect changes of chronic micro vascular ischemic disease, and often develop in persons with type 2 diabetes [1]. Higher WML load is associated with risk factors for cardiovascular disease (CVD), such as multiple sclerosis, diabetes, hypertension and tobacco use. WML predict an increaded risk of stroke, dementia, cognitive decline and death. Quantitative analysis of WML in large clinical trials may improve diagnosis and prognosis of the diseases affecting cerebral white matter. White Matter Lesions (WML) refer to areas of high intensity observed on $\mathrm{T}_{2}$ - weighted or Fluid Attenuated Inversion Recovery (FLAIR) Magnetic Resonance Imaging (MRI) within cerebral white matter.
Manual delineation of WML boundaries from FLAIR MRI is laborious and tedious. Hence, WML grading is often done using a semi-quantitative subjective 10 points rating scale. However, this method suffers from poor interrater variability. Tools for automated white matter lesion segmentation are now becoming available but no gold standard has been established. Recently, two WML segmentation algorithms [1], [2] have received attention. The first is an automated tool designed for detecting FLAIR hyper-intense WML in MS proposed by Schmidt et al [2]. It first determines the three cerebral tissue classes (Gray Matter (GM), White Matter (WM) and Cerebro-Spinal Fluid (CSF)) from the $T_{1}$ - weighted images, then estimates the distribution of FLAIR intensities for each tissue class separately, and finally detects FLAIR hyper-intense outliers within each tissue class which are interpreted as lesion belief. This method iteratively computes the lesion belief by voxel-wise evaluation of the likelihood of belonging to gray or white matter against the likelihood of belonging to lesions. A Hidden Markov Random Field (HMRF) based segmentation model along with prior knowledge on WM location is incorporated. However, the HMRF model suffers from high computational overhead. Also, a major challenge is to design the neighborhood system of the HMRF model characterizing the patient-specific cerebral tissue structures.

The second WML segmentation algorithm, developed by Lao et al. [1] first extracts local features from four multiparametric $\left(\mathrm{T}_{1}\right.$ - weighted, $\mathrm{T}_{2}$ - weighted, PD (Proton Density)- weighted and FLAIR) MRI sequences. Then a support vector machine classifier is used to classify $3 \mathrm{D}$ structural MRI voxels into WML and non-WML classes. A major challenge of voxel based classification is to handle skewed distribution of positive and negative examples (number of voxels belonging to WML is substantially smaller than the rest of brain voxels). This leads to a natural bias of classification towards healthy tissues. They implement adaptive boosting prior to Support Vector Machine(SVM) to reduce the number of negative examples (healthy tissues in this case) by selecting only those samples that were misclassified in the first few iterations of boosting. Samples 
that were misclassified in boosting iterations are considered good representative samples of the respective classes for classification purposes since they are relatively difficult to classify. SVM models built on training these hard examples presumes that it would classify the rest of the relatively easier samples correctly. However, this sampling strategy might lead to over fitting. In addition, MRI data including all of these four modalities are not always available in all studies and training on using fewer than four modalities might degrade the expected performance of their model. For voxel based classification, it is also very difficult to incorporate suitable neighborhood information characterizing actual brain structures.

Here we present a new hierarchical level set [3] based curve evolution technique for WML segmentation that overcomes the difficulties encountered in voxel based segmentation models described above. Curve evolution techniques combine all sequential tasks associated with other segmentation techniques such as voxel classification based segmentation or edge detection methods into a variational framework and thus reduces the combined errors accumulated at each stage of the segmentation method. Typically a level set creates two partitions. For multiple segmentation, Coupled Level Sets (CLS) are used [4]. However, CLS can create only $2^{n}$ number of partitions where $n$ is an integer. Creating the number of partitions that are not a power of two using CLS is often computationally expensive. It creates the nearest $2^{n}$ partitions and then it requires post processing (region merging or splitting the partitions) to make the required number of partions. We implement a novel hierarchical level set that works based on a divisive (top - down) approach. We minimize the energy functional for HLS by maximizing the between class variance. The proposed HLS first implements a level set on an image creating two partitions. Then the goodness (separability) of the partition is measured by the ratio of variance between the partition to the total variance [5]. If the ratio exceeds a predefined threshold value then the partition is accepted. Then each partition is divided by the level set function if the separation criterion is satisfied. Thus the proposed HLS segments an image into nearly homogeneous regions (low intensity variance). Then we classify the homogeneous regions into WML and non-WML classes by Adaptive-Regularized Boosting (ARBoost) [6]. AR-boost selects important features from a set of regional features such as intensity, variance, entropy etc. for WML classification. AR- Boost proposes a loss function that incorporates a regularization term into the exponential loss function. This proposed regularization assigns more weight to the misclassified samples at each stage of the boosting iteration resulting in early convergence of boosting. AR - Boost allows the user to select optimal values for the weights associated with misclassified samples through cross validation.

In this work, we make the following major contributions:
(1) We introduce a novel Hierarchical Level Set (HLS) that does not require estimation of the number of levels in an image. (2) We employ an energy functional for HLS that maximizes the variance between two partitions. We first maximize the between class variance into the level set framework. (3) HLS is computationally less expensive and more initialization independent than coupled-level sets. (4) AR-Boost employs a regularization term in the exponential loss function that provides more weight to the misclassified samples at each iteration during boosting that leads to early convergence. (5) AR-Boost allows the user to select optimal weights over Adaboost through cross validation. (6) The proposed framework is evaluated for WML segmentation from FLAIR MRI in a cohort of diabetic subjects and experimental results demonstrate that it outperforms other state-of-the-art techniques.

\section{RELATED WORK}

The level set method [3] is one of the most successful tools for capturing boundaries of desired objects or tracking interfaces in an image. To capture boundaries or track interfaces, the level set function describing the boundaries or interfaces is defined implicitly by a partial differential equation. The Energy functional is minimized by solving Euler-Lagrange Partial Differential Equations (PDE) and the level set function defining the boundary of the objects is obtained by solving the PDE numerically using a gradient descent method. The level set based PDE transforms the Euler Lagrange equations to a curve evolution technique where curve evolution starts from random initialization (naïve curve such as circles, rectangles etc.) and converges on the boundary of target objects. Typically level sets create 2 partitions. Multi-phase level sets have been proposed in the literature to capture multiple partitions. Multi-phase models can segment $2^{n}$ phases/regions of the image, where $n$ is the number of level set functions. Thus, the multi-phase model partitions the image into more than the number of desired regions when the expected number of regions is not a power of two. In this case the level set collapses for the redundant regions and the redundant regions become empty.

The hierarchical approach proposed by Tsai et al. [3] and Jeon et al. [4] relieves the complexity of multi-phase level sets that can segment the required number of regions. The hierarchical level set method proposed by Tsai et al. requires user intervention at each stage of segmentation to decide whether any subregion needs to be segmented further. Jeon et al. proposed a hierarchical level set method that successively segments image subregions automatically at each stage of the segmentation technique using a decision criterion based on the intensity variance across the current subregion. The region having larger variation becomes the target of the next step of the hierarchy. The segmentation stops when a specified number of levels has been reached. 
However, a major challenge in segmentation analysis is the estimation of the optimal number of levels. In addition, the regions having relatively lower variation at any hierarchy may require splitting if it is not a nearly homogeneous region.

\section{Proposed Algorithm}

The proposed Methodology for WML segmentation is shown in Figure 1. The method takes three dimensional T1 and FLAIR MRI images of a patient as input and returns the the WML found on FLAIR images. Broadly, we divide the proposed tasks for WML segmentation into three sequential subtasks: (a) Preprocessing performed using the VBM8 toolbox of Statistical Parametric Mapping (SPM) [7]; (b) Hierarchical segmentation performed by HLS and (c) Classification conducted by AR - Boost [6]. Details of these three subtasks are provided below.

\section{A. Preprocessing}

We use SPM8 and the VBM8 toolbox [7] for preprocessing T1 and Flair 3-D structural MRI. We first apply the VBM8 segmentation algorithm on T1 images to classify the cerebral tissues into three classes (Gray matter (GM), White Matter (WM) and CerebroSpinal Fluid (CSF)). The VBM8 segmentation algorithm generates patient - specific GM, WM and CSF masks. Then we fill the small holes of WM mask through morphological hole filling operation. For FLAIR images we remove the skull and then perform bias correction using SPM8. Then we register the preprocessed (skull stripped and bias corrected) FLAIR image as shown in Figure 1 with WM mask generated from the T1 images using a Mutual Information (MI) based registration algorithm implemented in the VBM8 toolbox. We exclude the cerebral tissues outside the WM mask from the FLAIR images for WML segmentation.

\section{B. Hierarchical Level Sets (HLS)}

First we explain the proposed variance based energy functional used into levelset framework and then elaborate on the proposed Hierarchical Level Set (HLS).

1) Variance based Energy Functional: Let us define $\Omega$ to be a bounded open subset of $\mathbb{R}^{2}$, with $\partial \Omega$ its boundary. Let us define the evolving curve $C$ in $\Omega$, as the boundary of an open subset $\omega$ of $\Omega$ (i. e. $\omega \subset \Omega$, and $C=\partial \omega$ ). Thus inside $(C)$ denotes the region $\omega$, and outside $(C)$ denotes the region $\Omega \backslash \omega$. Let $I: \Omega \rightarrow \mathbb{R}$ be a given image. Our method is the minimization of an energy based segmentation. Similar to Otsu Threshold [5], we try to maximize the variances between inside and outside regions of the curve $C, \sigma_{b}^{2}$, i.e., $\sigma_{b}^{2}$ is maximized if the curve $C$ lies on the interface of two distinct regions. Now, $\max \left(\sigma_{b}^{2}\right)=\min \left(-\sigma_{b}^{2}\right)=$

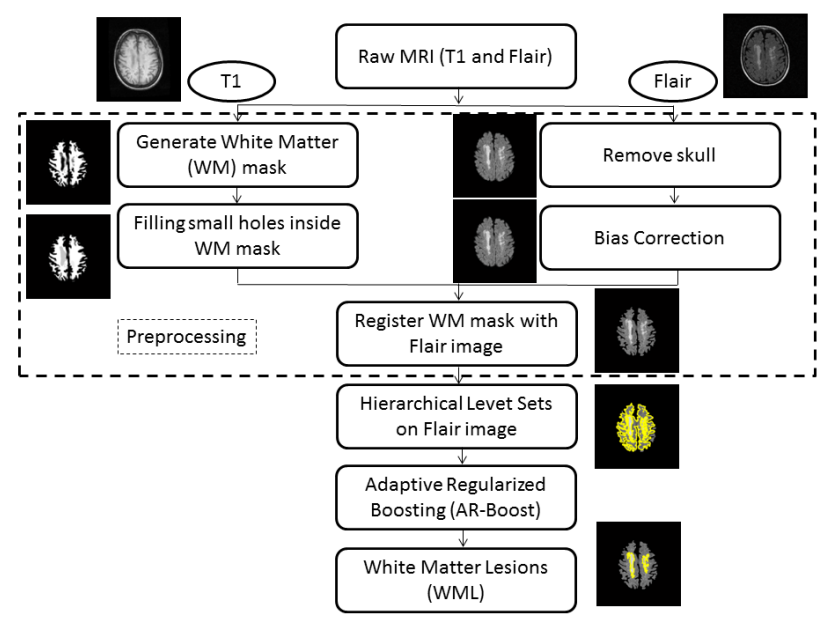

Figure 1. Proposed methodology for WML segmentation.

$\min \left(-N_{1} N_{2}\left(\mu_{1}-\mu_{2}\right)^{2} / N\right)$. Now, the energy functional $E$ which is the logarithm of $\max \left(\sigma_{b}^{2}\right)$, i. e.,

$$
E=-\left(\log N_{1}+\log N_{2}+2 \log \left(\mu_{1}-\mu_{2}\right)-\log N\right)
$$

If we consider that the image consists of two regions, the segmentation curve $C \subset \Omega$ can be represented by the zero level set of a Lipschitz function $\phi: \Omega \rightarrow \mathbb{R}$, where $C=(x, y) \mid \phi(x, y)=0$, such that $\phi(x, y, t)= \begin{cases}=0 & \text { if }(x, y) \text { is on } C, C=\partial \omega \\ >0 & \text { if }(x, y) \in \text { inside }(C) \text {, inside }(C)=\omega \\ <0 & \text { if }(x, y) \in \text { outside }(C) \text {, outside }(C)=\Omega \backslash \omega .\end{cases}$ Using heaviside function, $H(x)$ and the one-dimensional Dirac measure $\delta, H(x)=\left\{\begin{array}{l}1 \quad \text { if } x \geq 0, \\ 0 \quad \text { otherwise. }\end{array}\right.$ and $\delta(z)=\frac{d}{d z} H(z), N_{1}, N_{2}, \mu_{1}$ and $\mu_{2}$ can be defined by, $N_{1}=\int_{\Omega} H(\phi) \partial x \partial y, N_{2}=\int_{\Omega}(1-H(\phi)) \partial x \partial y$, $\mu_{1}=\frac{\int_{\Omega} I H(\phi) \partial x \partial y}{\int_{\Omega} H(\phi) \partial x \partial y}, \quad \mu_{2}=\frac{\int_{\Omega} I(1-H(\phi)) \partial x \partial y}{\int_{\Omega}(1-H(\phi)) \partial x \partial y}$.

The curve evolution equation can be obtained by minimizing the energy functional (1) with respect to $\phi$, and we deduce the associated Euler-Lagrange equation for $\phi$ as,

$$
\frac{\partial \phi}{\partial t}=\delta(\phi)\left[\frac{1}{N_{1}}-\frac{1}{N_{2}}+\frac{2}{\left(\mu_{1}-\mu_{2}\right)}\left(\frac{I-\mu_{1}}{N_{1}}-\frac{I-\mu_{2}}{N_{2}}\right)\right]
$$

Where, $\phi(0, x, y)=\phi_{0}(x, y)$ in $\Omega, \frac{\delta(\phi)}{|\nabla \phi|} \frac{\partial \phi}{\partial \vec{n}}=0$ on $\partial \Omega$. $\vec{n}$ denotes the exterior normal to the boundary $\partial \Omega$, and $\partial \phi / \partial \vec{n}$ denotes the normal derivative of $\phi$ at the boundary. $\phi(0, x, y)=\phi_{0}(x, y)$ defines the inital contour.

2) Hierarchical Level Set (HLS): Pseudocode for the proposed HLS algorithm are illustrated in Algorithm 1. In this work, we propose a novel approach for Hierarchical Level Set (HLS) method that automatically partitions the image into a number of nearly homogeneous (low intensity variance) regions 1 . Level set solver (line 7 in 


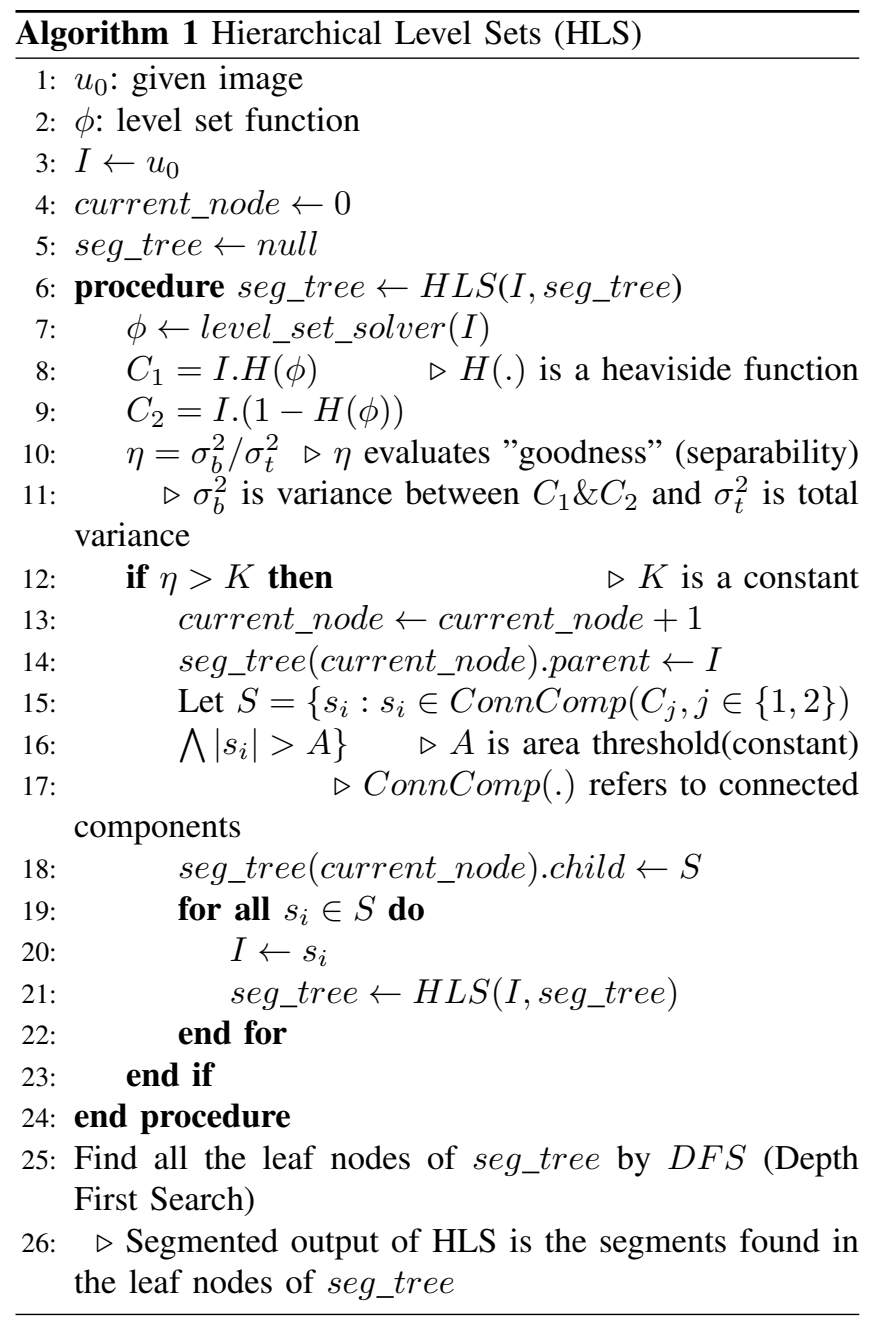

Algorithm 1) minimizes variance minimization based energy functional(equation (1) and (2)) as dicussed in subsection III-B1. Two advantages of the proposed method over its competitors are mentioned here. Firstly, unlike Jeon et al. [4], our method does not require apriori knowledge about the number of inherent levels in an image. Estimating the optimal number of levels in an image is difficult. Secondly, at each level of the hierarchy we create two partitions using a level set for each candidate connected component separately only if the value of the "goodness" (or separability) [5] of creating two partitions exceed a threshold value (line number 10 - 12 of Algorithm 1) that can be easily determined through cross validation. Discriminant criterion $(\eta$ in line number 10 of Algorithm 1) [5] is the ratio of variance between two partitions and total variance that measures the separability between two partitons. We have used the value of $K$ (in line number 12 of Algorithm 1) as 0.3 in our experiment. Thus, HLS partitions the image into a number of nearly homogeneous regions at the end of the hierarchy and doesn't need to segment further. On the contrary, Jeon $e t$ al. [4] only create partitions if the regions have higher variance at each level of hierarchy. Regions having lower variance may need to be split again and it is not certain that all the output segments at the end of the hierarchy appear as nearly homogeneous regions.

Then we classify the nearly homogeneous regions found from HLS into WML and non-WML classes using AR Boost.

\section{Adaptive Regularized Boosting (AR-Boost)}

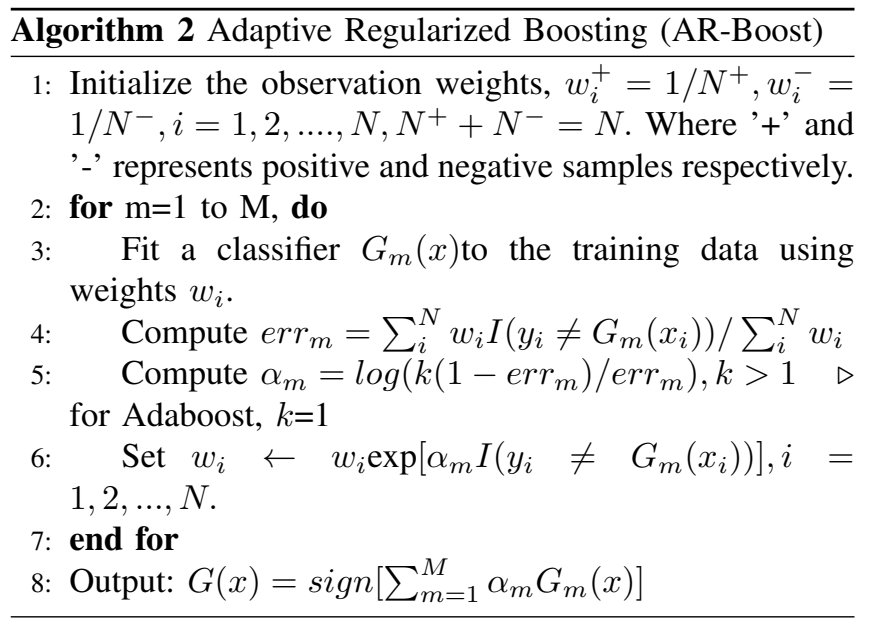

We compute different features such as average intensity, entropy, variance, etc. for each region found by HLS. We use Adaboost (variant of boosting) for selecting important features [8]. On training images, we classify regions found by HLS as positive examples that show high overlap ratio (higher value of Dice Coefficient [6]) with expert delineated WML and consider other regions found by HLS as negative examples. We employ decision stump (threshold) [8] as the underlying weak classifier. At test phase, we first apply HLS to partition the images into a number of segments and we compute the values of the important features chosen by boosting during training for each region. Then we multiply them with the weights associated with the features chosen by boosting during the training phase and subsequently add them to form a strong classifier, $G(x)=\operatorname{sign}\left(\sum_{m=1}^{M} \alpha_{m} G_{m}(x)\right)$, where, $\alpha_{m}$ is the weight associated with weak classifier $G_{m}($.$) . If the sign of the$ response of the strong classifier for a region is positive then it is only classified as WML.

For classification, Adaboost minimizes an exponential loss function [8]: $L(y, f(x))=\exp (-y f(x))$, where $y$ is the response and $f$ is the prediction. Figure 2: left shows different loss functions for two - class classification as a function of the margin $y . f(x)$. Misclassfication loss $L(y, f(x))=I(y \cdot f(x)<0)$, gives unit penalty for negative margin values, and no penalty for all positive margin values. 

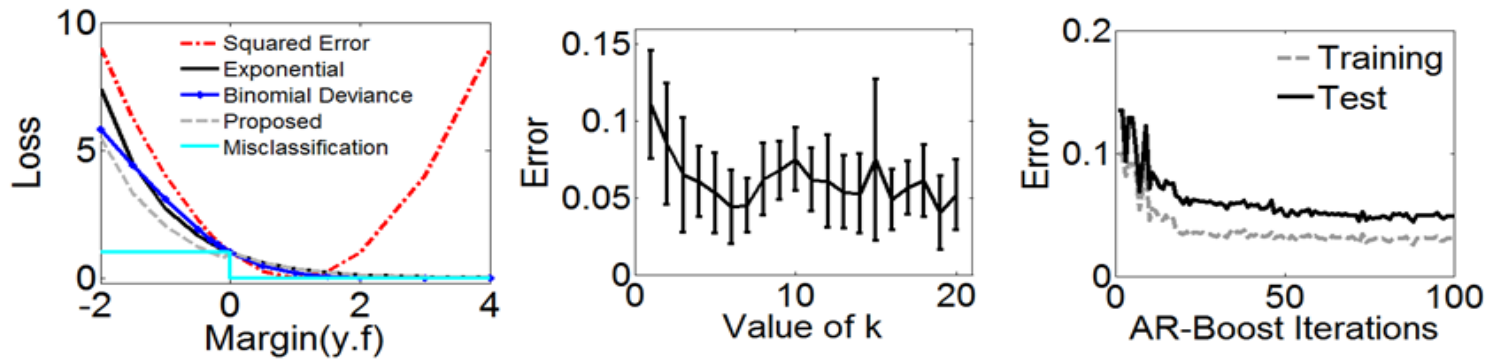

Figure 2. Left: Loss functions for two-class classification. Middle: 5-fold cross validation with standard error bars. Right: Misclassification error over number of iterations for $k=6$.

Squared-error loss $L(y, f(x))=(y-f(x))^{2}$, is not a good alternate for misclassification error. Figure 2 shows that squared-error loss is not a monotone decreasing function of increasing margin $y f(x)$. For margin values $y_{i} f\left(x_{i}\right)>1$ it increases quadratically, thus adopting increasing influence (error) on observations that are correctly classified with increasing certainty, which nullifies the relative influence of those incorrectly classified $y_{i} f\left(x_{i}\right)<0$. It is noted here that a monotone decreasing criterion serves as a better alternate loss function. Both binomial deviance $L(y, f(x))=\log (1+\exp (-2 y f(x)))$, and exponential loss can be observed as monotone continuous approximations to misclassification loss as shown in Figure 2: left. The penalty associated with binomial deviance increases linearly for large increasingly negative margin, whereas the exponential loss function penalizes exponentially for large increasingly negative margin ones than they reward increasingly positive ones [8].

One drawback of this exponential loss function is that it incurs substantial misclassification error rate as the penalty increases exponentially for large increasing negative margin due to outliers [8]. To address this problem, we propose a novel loss function: $L(y, f(x))=\exp (-y f(x)+\lambda \mid y-$ $G(x) \mid)$, where $\lambda<0$ and $G($.$) is the prediction of the weak$ classifier chosen at the current stage. We have introduced one additional term to the exponential loss function that acts as a regularizer. At any boosting iteration, the proposed loss function is the same as the existing loss function if the misclassification error rate at the current stage is zero (proposed term vanishes when $\lambda=0$ ). The penalty associated with the proposed loss function is less than that of the exponential one, if the misclassification error rate at the current stage is not equal to zero (shown in Figure 2: left where loss is plotted against a function of the classification margin y.f). This modification leads to more weight to the misclassified samples at any iteration and it enforces the misclassified samples to classify correctly in the next iteration. One additional advantage of this proposed loss function is that the user can adjust the amount of penalty for negative margins after observing the classifier performance over a training data set. Accordingly, we determine the value of $\lambda$ through cross validation ( $\lambda$ is a function of $k$ (the value of $k$ is determined experimentally). We derive a modified Adaboost algorithm that we call Adaptive Regularized Boosting(AR-Boost) as illustrated in Algorithm 2 by minimizing the proposed loss function (The derivation is shown in [6]). Our modified Adaboost finds the feature weight, $\alpha_{m}=\log \left(k\left(1-e r r_{m}\right) / e r r_{m}\right), k \geq 1$, (line number 5 of Algorithm 2) where, for the existing Adaboost algorithm the value of $k$ is always 1 . This leads to the weights associated with misclassified observations at any stage being $k$ times as much as the existing Adaboost [6]. The value of $k$ for AR - Boost is determined by cross-validation as shown in Figure 2: Middle and is discussed in the next section. Our proposed term in the existing loss function acts as a regularizer in the boosting framework. Our method can adaptively adjust the effects of regularization in the boosting framework by selecting the proper value of $k$ from the training data set as shown in Figure 2 : Middle. Figure 2 : Middle shows the misclassification error is minimum for $\mathrm{k}=6$. Figure 2 : Right shows the misclassification error over AR-Boost iterations.

\section{Results AND Discussions}

\section{Subjects and Magnetic Resonance Imaging}

Images used in this study were collected from ongoing studies investigating white matter changes in diabetes at Wake Forest. We obtained a sample of 50 randomly selected control subjects, and 50 diabetic patients.We used a 3D structural T1-weighted sequence, and a 3D structural FLAIR sequence.

\section{Qualitative and Quantitative Evaluation}

We compare our proposed method for WML segmentation with SVM method proposed by Lao et al. [1], HMRF method proposed by Schmidt et al [2] that is available in SPM8 Lesion Segmentation Toolbox (LST) [9] and a thresholding based segmentation algorithm followed by morphological operations. Results of these methods on Flair images of three different patients are demonstrated in Figure 3. The SPM8 LST [9] based WML segmentation maps were generated for each subject using 20 thresholds (k) 

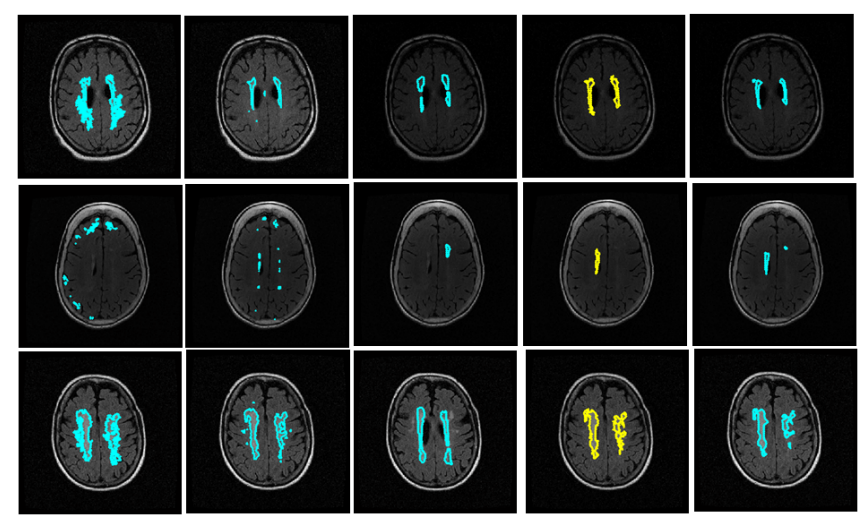

Figure 3. Results of Different Methods. Left column:Global Thresholding; Second column from Left:SVM method proposed by Lao et al. [1]; Third column from Left:HMRF method proposed by Schmidt et al [2]; Second column from Right: Proposed Methodology; Right column: Manual Segmentation.
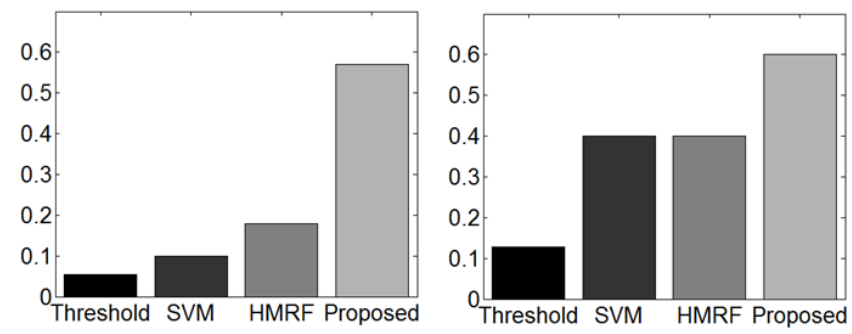

Figure 4. Left: Dice Coefficient (DC) and Right: Pratt's Figure of Merit (PFOM).

ranging from $0-1$ at .05 increments with the best threshold chosen through cross validation [8]. Ground Truth (GT) was determined using manual segmentation of the FLAIR images by an experienced reader. The performances of our proposed method, SVM, HMRF or LST, and Thresholding were compared against the manual segmentations using the Dice Coefficient (DC) [6] and Pratt's Figure of Merit (PFOM) [6]. Average DC and PFOM for all of these methods on 100 subjectes are illustrated in Figure 4. The values of DC and PFOM lie beween 0 and 1. Higher DC indicates higher overlap ratio between segmented output and actual objects found in GT. Higher PFOM refers to less distance between the edges of segmented objects and actual objects in GT. Higher DC and PFOM in Figure 4 demonstrates superiority of the propsed algorithm (HLS followed by AR-Boost) over SVM, LST and thresholding. The proposed technique is able to trace irregular shaped and less intense WML more accurately than other methods as demonstrated in Figure 3.

\section{CONCLusions}

We have demonstrated a novel automated Hierarchical Level Set (HLS) method and Adaptively Regularized Boosting (AR- Boost) classifier that outperforms two state-of-the- art algorithms, [1] and [2] or [9] for WML segmentation. Unlike other hierarchical level set methods in [3], [4], the proposed HLS does not require anticipating the inherent number of levels in an image. HLS is computationally faster than coupled level sets since it doesn't create unnecessary regions like coupled level sets. HLS is more initialization independent than coupled level set since if the curve evolution starts away from the desired interface, the divisive (top-down) partitioning criteria helps to initialize the level set near the desired boundary at the lower/lowest level of the hierarchy. The proposed HLS takes a divisive (top-down) approach and iteratively partitions the image into a number of nearly homogeneous regions (low intensity variance). Adaptive Regularized Boosting (AR-Boost) classifies the segments into WML and non-WML classes. AR-Boost exploits a modified exponential loss function that assigns more weight to the misclassified samples than adaboost at each stage of the boosting iteration. This forces misclassified samples to be classified correctly at the next iteration and leads to early convergence. Users can choose optimal values of the weights using cross validation during training. The proposed algorithm can delineate WML using FLAIR images and uses $T_{1}$ - weighted images to remove false positives residing outside the WM mask. This proposed technique may be useful for investigating a wide range of cerebrovascular diseases that affect cerebral white matter.

\section{REFERENCES}

[1] Z. Lao, D. Shen, D. Liu, A. F. Jawad, E. R. Melhem, L. J. Launer, R. N. Bryan, and C. Davatzikos, "Computer-assisted seg. of wml in 3D MR images using svm," Acad. Rad., vol. 15, no. 3 , pp. 300-313, 2008.

[2] P. Schmidt, C. Gaser, M. Arsic, D. Buck, A. Frschler, A. Berthele, and et al., "An automated tool for detection of flair-hyperintense white-matter lesions in ms," NeuroImage, vol. 59, no. 4, pp. $3774-3783,2012$.

[3] A. Tsai, A. Yezzi, and A. S. Willsky, "Curve evolution implemen. of the mumford-shah func.l for image seg., denois., interp., and mag." IEEE Trans. on IP, pp. 1169 - 1186, 2001.

[4] M. Jeon, M. Alexander, W. Pedrycz, and N. J. Pizzi, "Unsupervised hierarchical image segmentation with level set and additive operator splitting," PRL, pp. 1461 - 1469, 2005.

[5] N. Otsu, "A Threshold Selection Meth. from Gray-level Hist." IEEE Transactions on Sys., Man and Cyb., pp. 62-66, 1979.

[6] B. N. Saha, N. Ray, and H. Zhang, "Automating snakes for multiple objects detection," in $A C C V, 2010$, pp. 39-51.

[7] http://dbm.neuro.uni-jena.de/vbm.html.

[8] T. Hastie, R. Tibshirani, and J. Friedman, The Elements of Statistical Learning: Data Mining, Inference, and Prediction. Springer, Second Edition, 2009.

[9] http://www.applied-statistics.de/lst.html. 